\title{
Extragonadal Germ Cell Tumors in Children and Adolescents - a 20 Year Study
}

Felice FAIZAL ${ }^{1}$, Alka KALGUTKAR², Vandana DAHAKE

\begin{abstract}
Germ cell tumors (GCTs) are rare neoplasms which are seen in all age groups and both sexes. GCTs in the pediatric age group show considerable variation from their adult counterparts in relation to site, histological type, clinical and radiological features and response to treatment. Extragonadal gcts are much more common in the pediatric age group with the most common sites being sacrococcygeal region, head and neck, retroperitoneum and mediastinum. Unusual sites like the vagina and urinary bladder have also been reported. Therefore, the varied presentations and different treatment options should be considered
\end{abstract}

Keywords: germ cell tumors, extragonadal germ cell tumors, sacrococcygeal teratomas, mediastinal teratomas, pediatric germ cell tumors.

\section{INTRODUCTION}

Germ cell tumors (GCTs) are a complex group of neoplasms that can be benign or malignant. Despite their heterogeneity they are all presumed to arise from the totipotent primordial germ cells. Seen among all age groups, they represent $16 \%$ of all tumors amongst adolescents and $3.5 \%$ of all tumors in children ${ }^{1}$. The GCTs in the children show considerable variation from their adult counterparts with respect to site, histological types and response to treatment ${ }^{2}$. Extragonadal GCTs (EGCTs) are much more common in children which prompted us to take up this study.

\section{MATERIALS AND METHODS}

\section{Ethics}

The study process was reviewed and passed by the institutional review board

\section{Study design}

All specimens of extragonadal GCTs in patients less than 18 years of age received over a period of 20 years were included in this study. Clinical details and investigations were obtained from the records retrospectively and prospectively. Extensive grossing of specimens and routine processing with $\mathrm{H}$ and $\mathrm{E}$ staining was done on all specimens. Special stains were done wherever necessary.

\section{RESULTS}

A total of 110 GCTs (gonadal + extragonadal) were received during the study period. The annual rate of occurrence of GCTs ranged from $0.4 \%$ to $2.58 \%$ amongst all pediatric specimens with an average of $1.21 \%$.

Of the 110 GCTs, 48 (43.6\%) were in the extragonadal regions. The most common sites were sacrococcyx
1 Department of Pathology, Army Medical Corps, New Delhi, India ${ }^{2}$ Department of Pathology, LTMMC, Mumbai, India
Corresponding author:

Felice FAIZAL, A 201, Kailash towers, IGNOU road, New Delhi, zip code: 110030 , India.

E-mail: flcfzl@gmail.com 
followed by head and neck, retroperitoneum and mediastinum. Distribution according to site, sex and age group is shown in Table 1.

\section{DISCUSSION}

GCTs are rare tumors which can occur at any site. In young children, the most common sites in decreasing order of frequency are ovary (26\%), coccyx (24\%), testis (18\%) and brain (18\%). Extragonadal GCTs are rare amongst all GCTs if we consider all age groups but their incidence is quite high in the pediatric population $^{2}$. Site wise distribution and salient features have been discussed in detail.

\section{Sacrococcyx}

GCTs of the sacrococcygeal region cases are diagnosed as an obvious mass at birth but can be detected prenatally. Endodermal sinus tumors (YST) are usually detected later in early childhood ${ }^{3}$.

In the present study there were twelve cases of GCTs in the sacrococcygeal and two cases in the presacral region. Of these 14 cases, twelve were teratomas and two were YSTs. $71 \%$ of the cases were seen in females. Female predominance and similar histological distribution has been seen in various studies ${ }^{4-6}$.

All the cases of benign sacrococcygeal teratomas (SCTs) in our study were treated with mass removal with coccygectomy, except in two cases, where only mass removal was done. The survival of benign SCTs was $90.6 \%$. A 5 day old female child with a large SCT measuring $13 \times 6 \times 6 \mathrm{~cm}$ was treated with mass excision with removal of coccyx but did not survive.

There were 3 malignant tumors in the sacrococcygeal region of which two were Yolk Sac Tumors. One was a 12 year old female with a tumor in the sacrococcygeal region and another was a 2 year old male child with a tumor in the presacral region. Both were given postoperative chemotherapy after mass removal but none of them survived.

One was a case of grade 2 Immature Teratoma in a 1 day old female child who was treated with mass excision with coccyx removal successfully.

The 5 year survival in malignant lesions in our study was $33 \%$ which is lower than that seen by Schropp et al. $(43 \%)^{6}$.

\section{Mediastinum}

Mediastinal GCTs are seen across all age groups and in both sexes. They constitute around 7\% of all GCTs in childhood. Benign tumors of the mediastinum are mostly detected on chest X-rays while the patient is asymptomatic. Symptoms if present, include cough, dyspnea, chest pain and mass effects like superior vena cava syndrome. Malignant mediastinal GCTs on the other hand are frequently symptomatic with cough and dyspnea, fever and weight loss. Sometimes patients may present with metastatic disease ${ }^{7}$.

We had 7 cases of mediastinal GCTs which accounted for $6.5 \%$ of all GCTs in the pediatric age group. Even though literature states no sex predilection, 71\% of mediastinal GCTs in our study were in males.

Histologically, five were Mature Teratomas and one case each was of an Immature Teratoma and Mixed malignant GCT. One case was diagnosed in a preterm male child which was still born and on autopsy, the tumor was diagnosed to be an Immature Teratoma.

All the cases except the stillborn child were treated with surgical excision alone and $100 \%$ survival was seen. This is in accordance with Craig who states that for benign mediastinal teratomas, complete resection results in near total cure rates and there is no role for chemotherapy or radiotherapy ${ }^{7}$.

\section{Head and neck}

Approximately $6 \%$ of all GCTs in children occur in the soft tissue of the head and neck region. The main extracranial head and neck locations are the soft tissue of lateral neck, orbit and nasopharynx in decreasing order of frequency ${ }^{8,9}$. We saw nine cases of GCTs in the head and neck region which accounted for $8.4 \%$ of all GCTs.

Of the 9 cases, three were in the cervical region, one was in the retro-orbital region, three were in the epignathic region and two were in the oropharyngeal and nasopharyngeal region. Equal incidence was seen in both sexes.

One case each of a Yolk Sac Tumor, Immature Teratoma and a Mixed malignant GCT were seen in our study, all three arising in the neck region. Rest were Mature Teratomas.

Orbital teratomas are rare congenital tumors whose presenting features include unilateral proptosis, stretched eyelids, elongation of the palpebral fissure or enlargement of the bony orbit. Vision may not be preserved because of involvement of the optic nerve. In literature fewer than $20 \%$ of the published cases were treated with methods aimed at preserving the eyeball. This was mainly due to the fear of malignancy but very 
Table 1: Extragonadal GCTs according to age group, sex and site

\begin{tabular}{|c|c|c|c|c|c|c|c|c|c|c|c|c|c|}
\hline \multirow[t]{2}{*}{ AGE GROUP } & \multirow[t]{2}{*}{ SEX } & \multicolumn{11}{|c|}{ SITE } & \multirow[t]{2}{*}{ TOTAL } \\
\hline & & 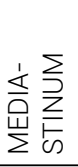 & $\begin{array}{ll} & x \\
0 & 0 \\
0 & 0 \\
0 & 0 \\
0 & 0 \\
\omega & 0\end{array}$ & 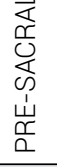 & 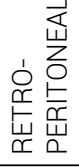 & 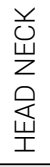 & 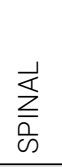 & 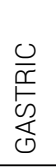 & $\begin{array}{l}\underset{\mathbb{\Psi}}{\rightleftarrows} \\
=\end{array}$ & 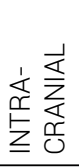 & 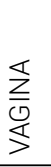 & 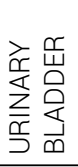 & \\
\hline \multirow{2}{*}{$0-1$ month } & M & 01 & 02 & - & 01 & 02 & - & - & - & - & - & - & 06 \\
\hline & $\mathrm{F}$ & - & 08 & 01 & 01 & 02 & 01 & - & - & - & - & - & 13 \\
\hline \multirow{2}{*}{ 1month-1 year } & M & 02 & 01 & - & - & 01 & - & 01 & - & - & - & - & 05 \\
\hline & $\mathrm{F}$ & 01 & - & - & 02 & 03 & - & - & - & - & 01 & - & 07 \\
\hline \multirow{2}{*}{ 1year-5 years } & M & - & - & 01 & 01 & - & - & - & 01 & - & - & - & 03 \\
\hline & $\mathrm{F}$ & - & - & - & - & - & - & - & - & - & - & - & 00 \\
\hline \multirow{2}{*}{ 5years- 10 years } & M & - & - & - & - & - & 02 & - & - & - & - & - & 02 \\
\hline & $\mathrm{F}$ & 01 & - & - & - & - & - & - & - & 01 & - & - & 02 \\
\hline \multirow{2}{*}{ 10years- 15 years } & M & 01 & - & - & - & - & - & - & - & 02 & - & - & 03 \\
\hline & $\mathrm{F}$ & - & 01 & - & - & - & - & - & - & 01 & - & - & 02 \\
\hline \multirow{2}{*}{ 15years-18 years } & M & 01 & - & - & - & 01 & - & - & - & - & - & - & 02 \\
\hline & $\mathrm{F}$ & - & - & - & 02 & - & - & - & - & - & - & 01 & 03 \\
\hline TOTAL & & 07 & 12 & 02 & 07 & 09 & 03 & 01 & 01 & 04 & 01 & 01 & 48 \\
\hline
\end{tabular}

few studies have reported malignant orbital teratomas $^{10-12}$. Therefore management of orbital teratomas should include orbit preserving methods unless malignancy is suspected.

In our study, orbital teratoma was seen in a 2 month old male child who presented with left orbital mass since birth. The mass was excised and the patient had light perception on the affected side.

There was a case of a non-midline Yolk sac tumor in a 6 month old girl child with Dandy walker syndrome, who presented with painless swelling in the left submandibular area ,extending from midline to posterior triangle to the thyroid cartilage. Serum AFP was markedly elevated and $\beta$ HCG was undetectable. Histopathology of the open biopsy confirmed diagnosis of Yolk sac tumor.

Association of Dandy Walker syndrome with facial hemangiomas, primary intracranial melanocytic and dermoid tumors is well known ${ }^{13}$. However no such association with a YST at this site has been reported and may represent a random association only.

Combination chemotherapy (Bleomycin, Etoposide, Paclitaxel) resulted in total regression of the tumor mass and AFP levels were undetectable after 6 cycles. The child was disease free after 8 months of follow up. However she succumbed to septicemia following infection of the ventriculo-peritoneal shunt which was placed to relieve the hydrocephalus.

\section{Retroperitoneum}

There were seven cases of retroperitoneal GCTs constituting $5.6 \%$ of all the cases. six (83.3\%) were Mature Teratomas and one case was of an Immature Teratoma (16.6\%).

Two cases of MTs in our study were masses in the abdomen that were suspected as fetus in fetu (FIF). 
Willis and Lord claim that presence of vertebral column, extremities and the orientation of organs around these structures are diagnostic of FIF ${ }^{14,15}$. To be called FIF the mass must demonstrate true organogenesis. Non-visualization of vertebral axis on radiography or CT Scan does not exclude FIF as the pathologist can confirm it on gross examination ${ }^{16}$.

One case was 900 gram retroperitoneal mass, which had presence of limb buds at the caudal and cephalic ends and a spine. Another case was of a 300 gram mass which had the presence of part of vertebral bone along with limb buds. Both these cases had organogenesis but no orientation around the central axis and hence were classified as fetiform teratomas instead of FIF.

All the cases of retroperitoneal GCTs were treated with surgical excision only. All cases of mature teratomas and fetiform teratomas survived whereas the newborn female with Immature Teratoma did not.

\section{Intracranial GCTs}

Intracranial GCTs vary in their geographic incidence and constitute 0.3 to $3 \%$ of primary CNS tumors. These tumors are generally found in the suprasellar region or the pineal region. $5-10 \%$ cases may show involvement of both the sites. Males tend to be more affected and a peak in the incidence near puberty has been observed. In suprasellar tumors, endocrine manifestations prevail whereas in pineal gland tumors,symptoms of raised intracranial tension are observed ${ }^{17}$.

Although the diagnosis in intracranial GCTs is suggested by CT \& MRI of brain, histopathological confirmation is necessary for a definitive diagnosis. Germinomatous GCTs show a very good prognosis with radiotherapy alone but the prognosis of non germinomatous GCTs is less favorable.

A total of four cases of intracranial GCTs were observed in the present study which accounted for 3.6\% of all GCTs. All cases presented in the age group of 5-15 years with equal incidence in both sexes.

Three were intracranial germinomas, of which two were in the pineal region and one in the suprasellar region. all three cases presented with varied symptoms and were treated differently.

One was a 12 year male who presented with visual disturbance with headache and was treated with surgical excision only.

Another case was of a 14 year old female child who presented with squint and diplopia and was treated with surgical excision and chemotherapy.
The third case was a 13 year old boy with multiple primary intracranial germinomas who presented with headache, vomiting, diminished vision $\&$ ataxia of 3 months duration. On examination, drowsiness, papilloedema and bilateral central scotoma were present. There was restriction of upward gaze but no nystagmus. $\beta$ HCG levels were markedly elevated to $994 \mathrm{mIU} / \mathrm{ml}$. CT scan revealed lesions in the suprasellar and in the quadrigeminal cistern, compressing foramen of Monro resulting in obstructing hydrocephalus. The child was treated with surgical excision followed by chemotherapy and radiotherapy.

The only case of the Mixed Malignant GCT was diagnosed in a 9 year old female which was earlier than those with germinomas. Non germinomatous intracranial GCTs present at an earlier age thereby suggesting a hormonal basis for germinomatous tumors which occur around puberty ${ }^{18}$. The child died soon after admission.

\section{Spinal teratomas}

Spinal teratomas are tumors of dysontogenetic origin which are situated in the spinal canal and can be extradural or intradural. Frequently they are associated with dysraphic effects like spina bifida and myelomeningoceles which has led some authors to believe that these may be a result of dysembryogenic mechanism and are not neoplastic ${ }^{19,20}$.

There were three cases of spinal teratomas in our study which accounted for $2.8 \%$ of all GCTs. Two of the cases were males. Associated ruptured meningomyelocele was seen in a newborn female child.

\section{Other rare extragonadal sites}

A single case of a gastric teratoma was seen in a 7 months old male child in our study which accounted
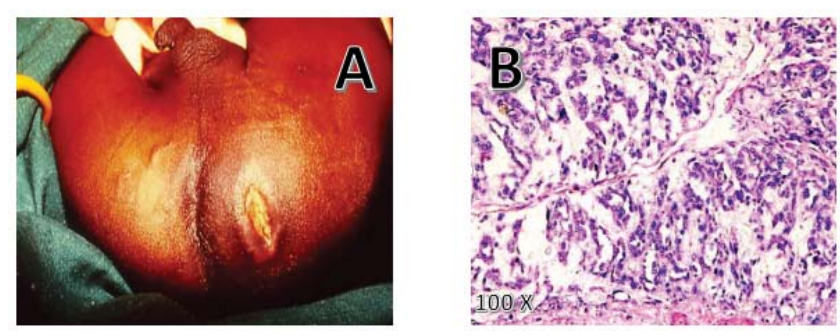

Figure 1. Presacral YST in a 6 month male A: Clinical picture of a male child showing a presacral tumor extending upto and ulcerating the overlying skin. B: H\&E stained section from the mass shows a reticular pattern of YST. 

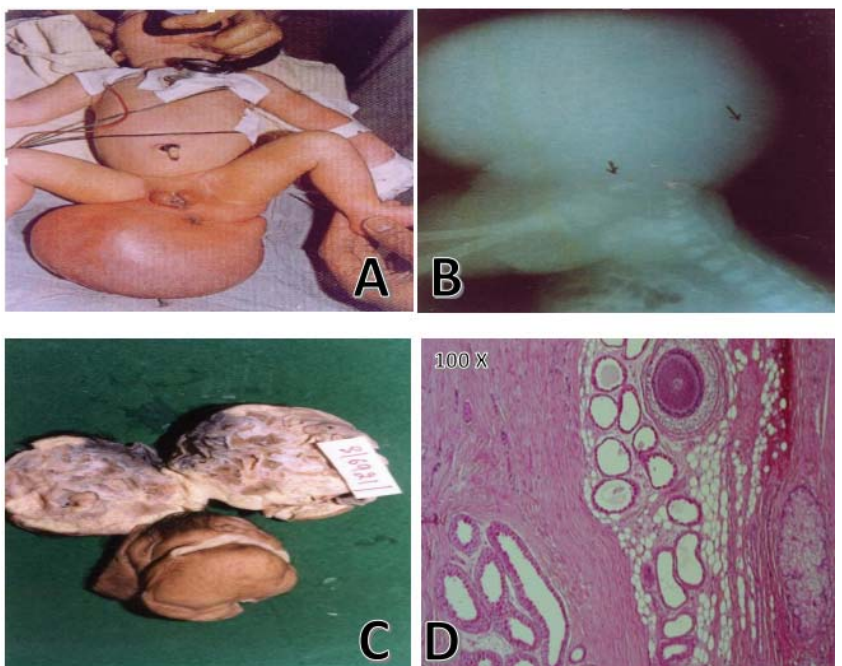

Figure 2. Sacrococcygeal teratoma in newborn. A: Clinical photograph of a female child with a large sacrococcygeal mass. B: X-ray of the mass showing bony structures and coccyx. C: Gross specimen of the resected mass showing a large tumor having a solid cystic appearance. D: H \& E stained section from mass shows areas of cartilage, fat, thyroid tissue and few intestinal glands.
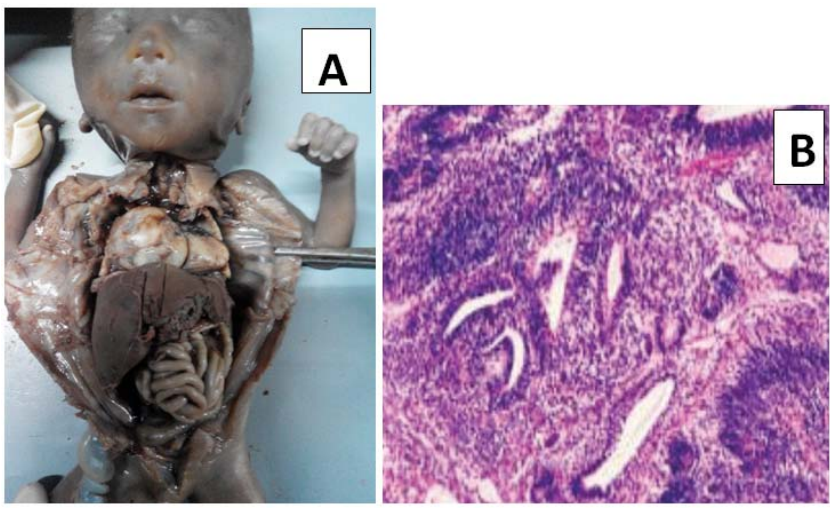

Figure 3. Pericardial immature teratoma in a stillborn A:in situ examination of a still born child during autopsy shows a large pericardial mass (arrow). B: H\&E stained section from tumor shows predominantly primitive neuroepithelium forming rosettes at places.
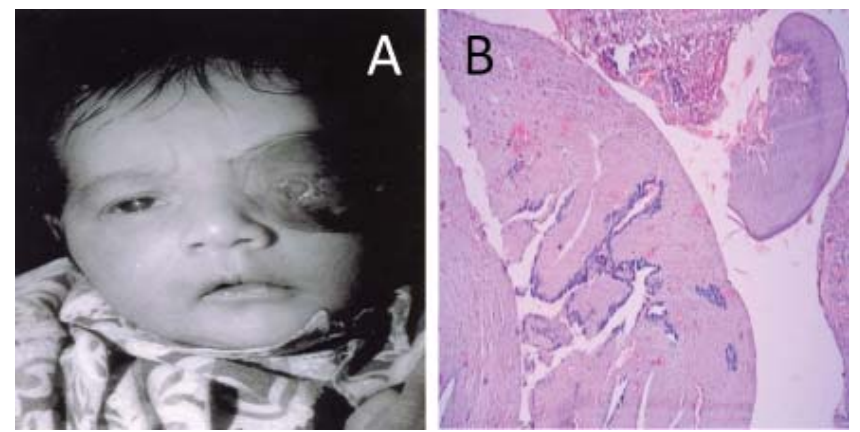

Figure 4. Orbital teratoma in a 2 month old child. A: Clinical photograph of a child showing an orbital mass replacing the entire orbit B:H\&E stained section from tumor shows mature glial tissue and immature neural epithelium forming rosettes at places.
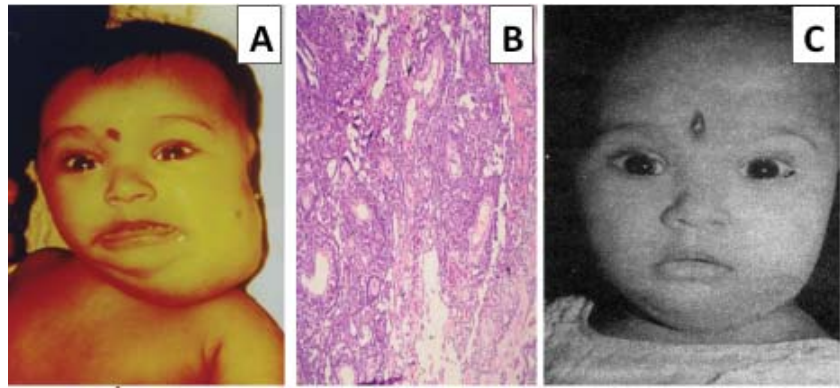

Figure 5. YST in the cervical region In a 6 month old girl child. A: Clinical photograph of a child with a large left sided neck mass. B: Histology of biopsy specimen shows reticular pattern of YST. C: The tumor regressed completely after 6 cycles of chemotherapy.
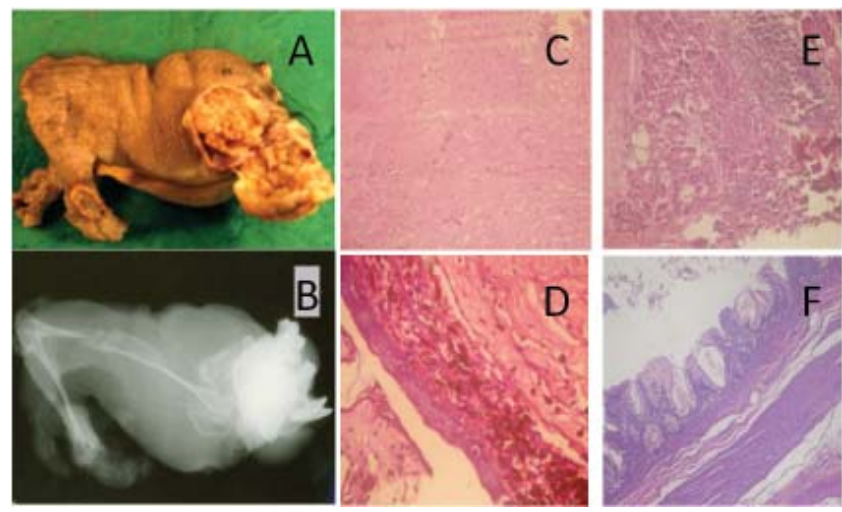

Figure 6. FETIFORM TERATOMA A: Large retroperitoneal teratoma with well-formed cranial and caudal ends and lower limb buds. B: $X$-ray of the mass shows well developed lower limb bones and rudimentary vertebrae. Presence of following organs were confirmed on histology: C: Mature brain tissue D: Choroidal tissue with pigmentation E: Thyroid follicles F: GIT showing all three layers.
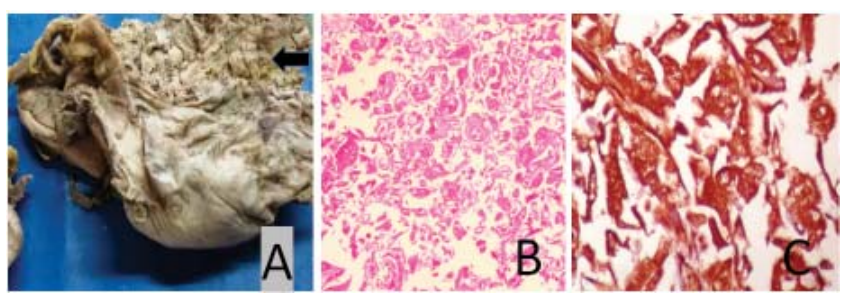

Figure 7. YST of the bladder A: A large friable mass in the bladder causing rupture of the bladder (arrow). B: H\&E section from mass showing a tumor composed of dyscohesive cells and hyaline globules at places. C: IHC for AFP. Positive. 
for $0.9 \%$ of all GCTs. Studies state that gastric teratomas account for less than $1 \%$ of all teratomas and majority of them present in the first year of age. $95 \%$ of those affected are males and excellent prognosis is seen after tumerectomy removing a thin rim of surrounding stomach wall ${ }^{21-23}$.

The occurrence of extra gonadal,intraperitoneal teratoma in infants and children, especially those arising from the mesentry and the mesocolon, are very rare. We had a single case in a $3 \frac{1 / 2}{2}$ year old male, who had a cyst attached to the ileum which presented clinically with abdominal pain. Cyst removal was done successfully.

One case was seen in the vagina in a 10 month old female child. Histologically the tumor was a grade 3 Yolk Sac Tumor. Surgical removal followed by chemotherapy was given and the patient survived.

An 18 year old female with complaints of abdominal pain, distention since 8 days and anuria since 2 days died soon after admission and no investigation was done. The case was sent for autopsy and a whitish friable mass involving the entire bladder \& causing rupture was identified.

$\mathrm{H}$ and $\mathrm{E}$ staining and positive IHC for AFP confirmed a diagnosis of YST. Since the histological exami-

\section{References}

1. Leslie Bernstein, Malcolm A. Smith, Lihua Liu, Dennis Deapen, Debra L. Friedman. Germ cell, Trophoblastic and other gonadal neoplasms, ICCC X.SEER pediatric monograph,NIH Pub. No. 99-4649,1999

2. U. Gobel, D. T. Schneider,G. Calaminus, R. J. Haas, P. Schmidt2 \& D. Harms on behalf of the GPOH MAKEI and the MAHO study groups.Germ-cell tumors in childhood and adolescence.Annals of Oncology 2000;1 1: 263-271

3. Keslar PJ, Buck JL, Suarez ES. Germ cell tumors of the sacrococcygeal region: radiologic-pathologic correlation. Radiographics. 1994 May; 14(3):607-20

4. Valdiserri, R. O. and Yunis, E. J. Sacrococcygeal teratomas: A review of 68 cases. Cancer.1981;48(1): 217-221

5. Mariko Yoshidaa, Kentaro Matsuokac, Atsuko Nakazawac, Makiko Yoshidad, Takeshi Inouee, Hiroshi Kishimotof, Masahiro Nakayamag, Emi Takabah, Minoru Hamazakih, Shigeaki Yokoyamai, Hiroshi Horiej, Mio Tanakaa, Kiyoshi Gomia, Youkatsu Ohamak, Hisato Kigasawal, Yoshihiro Kitanom, Hiroo Uchidan, Yutaka Kanamorio, Tadashi Iwanakab, Yukichi Tanaka, Sacrococcygeal yolk sac tumor developing after teratoma: A clinicopathological study of pediatric sacrococcygeal germ cell tumors and a proposal of the pathogenesis of sacrococcygeal yolk sac tumors. Journal of Pediatric Surgery. 2013 apr; 48(4):776-781

6. Kurt P. Schropp, Thom E. Lobe, Bhaskar Rao, Khaled Mutabagani, Gail A. Kay, Brian F. Gilchrist, Paul G. Philippe, E. Thomas nation of other organs including bilateral ovaries and uterus did not reveal any pathology, a diagnosis of primary YST of the urinary bladder was made.

Primary YSTs of the urinary bladder are extremely rare and only 2 cases have been reported in literature so $\operatorname{far}^{24,25}$.

\section{CONCLUSION}

Extragonadal GCTs are far more common in the pediatric population than in adults. They can be present in virtually any location but the most common sites are sacrococcyx, head and neck, retroperitoneum and mediastinum. The varied clinical presentations and sites can make the diagnosis difficult and awareness of the diverse morphological, clinical and radiological features of extragonadal GCTs is essential to ensure correct diagnosis and management.

Compliance with ethics requirements: The authors declare no conflict of interest regarding this article. The authors declare that all the procedures and experiments of this study respect the ethical standards in the Helsinki Declaration of 1975, as revised in 2008(5), as well as the national law. Informed consent was obtained from all the patients included in the study.

Boles. Sacrococcygeal teratoma: The experience of four decades. Journal of Pediatric Surgery.1992 aug;27(8):1075-1079

7. Craig $R$, Nichols. mediastinal germ cell tumors:clinical features and clinical correlates. Chest 1991;99:472-79

8. Lack EE. Extragonadal germ cell tumors of the head and neck region: review of 16 cases. Hum Pathol. 1985 Jan;16(1):56-64.

9. Louis P. Dehner, Alan Mills, Aleksander Talerman, Glenn F. Billman, Henry F. Krous, Charles E. Platz. Germ cell neoplasms of head and neck soft tissues: A pathologic spectrum of teratomatous and endodermal sinus tumors. Human Pathology.1990 march;21(3):309-318

10. Gnanaraj, Lawrence Skibell, Bentley C, Coret-Simon, Judith Halliday, William M.D, Forrest, Christopher ,DeAngelis, Dan D. Massive Congenital Orbital Teratoma. Ophthalmic Plastic \& Reconstructive Surgery.2005 nov;21(6):445-447

11. Schaal ST, Kampik A, Hasenfratz G, Boergen KP.Teratoma orbitae in the neonatal period. Klinische Monatsblatter fur Augenheilkunde .1988,;193(3):295-300

12. Eduardo J. C. Soares, Klaus Da Silva Lopes, José De Souza Andrade, Luiz Carlos Faleiro, and José Carlos Rezende Alve.Orbital malignant teratoma: A case report ,Orbit ,1983, Vol. 2, No. 4 , Pages 235-242

13. Kang SG1, Yoo DS, Cho KS, Kim DS, Chang ED, Huh PW, Kim MC. Coexisting intracranial meningeal melanocytoma, dermoid tumor, and Dandy-Walker cyst in a patient with neurocutaneous melanosis. Case report. J Neurosurg. 2006 Mar;104(3):444-7. 
14. Ibrahim Karaman, Derya Erdogan, Semire Ozalevli, Ayse Karaman, Y Hakan Cavusoglu, M. Kemal Aslan and Ozden Çakmak, Fetus in fetu: A report of two cases.J Indian Assoc Pediatr Surg. $2008 ; 13(1): 30-32$

15. Heifetz SA, Alrabeeah A, Brown BS, Lau H.Fetus in fetu: a fetiform teratoma.Pediatr Pathol. 1988;8(2):215-26.

16. Phatak S V, Kolwadkar P K, Phatak M S. Fetus in Fetu: A case report. Indian J Radiol Imaging. 2003;13:93-4

17. Roger J. Packer,Bruce H. Cohen,Kathleen Cooney. Intracranial Germ Cell Tumors. The Oncologist.2000;5(4) 312-320

18. Jennings MT, Gelman R, Hochberg F. Intracranial germ-cell tumors: natural history and pathogenesis. J Neurosurg. 1985 Aug;63(2):155-67.

19. S. Eneström, C. von Essen. Spinal teratoma. Acta Neurochirurgica. 1977;39(1-2): 121-126

20. Joseph L. Koen, M.D., Roger E. McLendon, M.D., and Timothy M. George, M.D.Intradural spinal teratoma: evidence for a dysem- bryogenic origin: Report of four cases.Journal of Neurosurgery.1998;89(5):844-851

21. D. K. Gupta,M. Srinivas,S. Dave,S. Agarwala,M. Bajpai,D. K. Mitra. Gastric teratoma in children.Pediatric Surgery International. 2000;16(5-6):329-332

22. Dr. Y. K. Sarin, L. D. Agarwal, V. N. Jhamaria, R. B. Goyal, R. Sharma, N. S. Shekhawat. Immature gastric teratoma.The Indian Journal of Pediatrics. 1997;64(6):896-898

23. Satge D, Auge B, Philippe E, Chenard MP, Sauvage P, Pusel J, Methlin G. Gastric teratoma in newborn children. Annales de Pediatrie.1990;37(4):235-241

24. Mui WH, Lee KC, Chiu SC, et al. Primary yolk sac tumour of the urinary bladder: A case report and review of the literature. Oncology Letters.2014;7(1):199-202

25. Taylor G, Jordan M, Churchill B, Mancer K. Yolk sac tumor of the bladder. J Urol. 1983 Mar;129(3):591-4. 
